\title{
THE UNIFIED RIEMANN-LIOUVILLE FRACTIONAL DERIVATIVE FORMULAE
}

\author{
R. C. SONI AND DEEPIKA SINGH
}

\begin{abstract}
In this paper, we obtain two unified fractional derivative formulae. The first involves the product of two general class of polynomials and the multivariable $H$-function. The second fractional derivative formula also involves the product of two general class of polynomials and the multivariable $H$-function and has been obtained by the application of the first fractional derivative formula twice and it has two independent variables instead of one. The polynomials and the functions involved in both the fractional derivative formulae as well as their arguments are quite general in nature and so our findings provide interesting unifications and extensions of a number of (known and new) results. For the sake of illustration, we point out that the fractional derivative formulae recently obtained by Srivastava, Chandel and Vishwakarma [11], Srivastava and Goyal [12], Gupta, Agrawal and Soni [4], Gupta and Agrawal [3] follow as particular cases of our findings. In the end, we record a new fractional derivative formula involving the product of the Konhauser biorthogonal polynomials, the Jacobi polynomials and the product of $r$ different modified Bessel functions of the second kind as a simple special case of our first formula.
\end{abstract}

\section{Introudction}

We shall define the Riemann-Liouville fractional derivative of a function $f(x)$ of order $\nu$ (or, alternatively, $-\nu$ th order fractional integral) [12, p.642, Eq.(1.1)] (see also [1, p.181; 8, p.49]) by

$$
{ }_{a} D_{x}^{\nu}\{f(x)\}= \begin{cases}\frac{1}{\Gamma(-\nu)} \int_{a}^{x}(x-t)^{-\nu-1} f(t) d t, & \operatorname{Re}(\nu)<0, \\ \frac{d^{q}}{d x^{q}}{ }_{a} D_{x}^{\nu-q}\{f(x)\}, & 0 \leq \operatorname{Re}(\nu)<q,\end{cases}
$$

where $q$ is a positive integer and the integral exists. For simplicity, the special case of the fractional derivative operator ${ }_{a} D_{x}^{\nu}$ when $a=0$ will be written as $D_{x}^{\nu}$. Thus we have

$$
D_{x}^{\nu} \equiv{ }_{0} D_{x}^{\nu}
$$

Also, $S_{n}^{m}[x]$ occurring in the sequel denotes the general class of polynomials introduced by Srivastava $[9$, p.1, Eq.(1)]

$$
S_{n}^{m}[x]=\sum_{k=0}^{[n / m]} \frac{(-n)_{m k}}{k !} A_{n, k} x^{k}, \quad n=0,1,2, \ldots,
$$

Received July 4, 2002; revised June 3, 2004.

2000 Mathematics Subject Classification. 26A33, 33C45, 33C60, 33C70.

Key words and phrases. Fractional derivative operator, general class of polynomials, multivariable $H$-function. 
where $m$ is an arbitrary positive integer and the coefficients $A_{n, k}(n, k \geq 0)$ are arbitrary constants, real or complex. On suitably specializing the coefficients $A_{n, k}, S_{n}^{m}[x]$ yields a number of known polynomials as its special cases. These include, among others, the Hermite polynomials, the Jacobi polynomials, the Laguerre polynomials, the Bessel polynomials, the Gould-Hopper polynomials, the Brafman polynomials and several others [16, pp.158-161].

The $H$-function of $r$ complex variables $z_{1}, \ldots, z_{r}$ was introduced by Srivastava and Panda [15]. We shall define and represent it in the following form [13, p.251, Eq.(C.1)]

$$
\begin{aligned}
& H\left[z_{1}, \ldots, z_{r}\right] \\
= & H_{P, Q: P^{\prime}, Q^{\prime} ; \cdots ; P^{(r)}, Q^{(r)}}^{0, N: N^{\prime}, N^{\prime} ; \cdots ; M^{(r)}}\left[\begin{array}{c|c}
z_{1} & \left(a_{j} ; \alpha_{j}^{\prime}, \ldots, \alpha_{j}^{(r)}\right)_{1, P}:\left(c_{j}^{\prime}, \gamma_{j}^{\prime}\right)_{1, P^{\prime}} ; \cdots ;\left(c_{j}^{(r)}, \gamma_{j}^{(r)}\right)_{1, P^{(r)}} \\
\vdots & \left(b_{j} ; \beta_{j}^{\prime}, \ldots, \beta_{j}^{(r)}\right)_{1, Q}:\left(d_{j}^{\prime}, \delta_{j}^{\prime}\right)_{1, Q^{\prime}} ; \cdots ;\left(d_{j}^{(r)}, \delta_{j}^{(r)}\right)_{1, Q^{(r)}}
\end{array}\right] \\
= & \frac{1}{(2 \pi \omega)^{r}} \int_{L_{1}} \cdots \int_{L_{r}} \phi_{1}\left(\xi_{1}\right) \cdots \phi_{r}\left(\xi_{r}\right) \psi\left(\xi_{1}, \ldots, \xi_{r}\right) z_{1}^{\xi_{1}} \cdots z_{r}^{\xi_{r}} d \xi_{1} \cdots d \xi_{r}
\end{aligned}
$$

where $w=\sqrt{-1}$,

$$
\begin{aligned}
\phi_{i}\left(\xi_{i}\right) & =\frac{\prod_{j=1}^{M^{(i)}} \Gamma\left(d_{j}^{(i)}-\delta_{j}^{(i)} \xi_{i}\right) \prod_{j=1}^{N^{(i)}} \Gamma\left(1-c_{j}^{(i)}+\gamma_{j}^{(i)} \xi_{i}\right)}{\prod_{j=M^{(i)}+1}^{Q^{(i)}} \Gamma\left(1-d_{j}^{(i)}+\delta_{j}^{(i)} \xi_{i}\right) \prod_{j=N^{(i)}+1}^{P^{(i)}} \Gamma\left(c_{j}^{(i)}-\gamma_{j}^{(i)} \xi_{i}\right)} \quad \forall i \in\{1, \ldots, r\} \\
\psi\left(\xi_{1}, \ldots, \xi_{r}\right) & =\frac{\prod_{j=1}^{N} \Gamma\left(1-a_{j}+\sum_{i=1}^{r} \alpha_{j}^{(i)} \xi_{i}\right)}{\prod_{j=N+1}^{P} \Gamma\left(a_{j}-\sum_{i=1}^{r} \alpha_{j}^{(i)} \xi_{i}\right) \prod_{j=1}^{Q} \Gamma\left(1-b_{j}+\sum_{i=1}^{r} \beta_{j}^{(i)} \xi_{i}\right)}
\end{aligned}
$$

The nature of contours $L_{1}, \ldots, L_{r}$ in (4), the various special cases and other details of the above function can be found in the book referred to above. It may be remarked here that all the Greek letters occurring in the left-hand side of (4) are assumed to be positive real numbers for standardization purposes; the definition of this function will, however, be meaningful even if some of these quantities are zero. Again, it is assumed that the various multivariable $H$-functions occurring in the paper always satisfy their apropriate conditions of convergence [13, pp.252-253, Eqs.(C.4-C.6)].

\section{Main Results}

Fractional derivative formula 1

$$
D_{x}^{\nu}\left\{x^{\rho}\left(x^{t_{1}}+\alpha_{1}\right)^{\sigma}\left(x^{t_{2}}+\alpha_{2}\right)^{\mu} S_{n}^{m}\left[e x^{u}\left(x^{t_{1}}+\alpha_{1}\right)^{v}\left(x^{t_{2}}+\alpha_{2}\right)^{w}\right]\right.
$$




$$
\begin{aligned}
& S_{n^{\prime}}^{m^{\prime}}\left[e^{\prime} x^{u^{\prime}}\left(x^{t_{1}}+\alpha_{1}\right)^{v^{\prime}}\left(x^{t_{2}}+\alpha_{2}\right)^{w^{\prime}}\right] \\
& \left.H\left[z_{1} x^{u_{1}}\left(x^{t_{1}}+\alpha_{1}\right)^{-v_{1}}\left(x^{t_{2}}+\alpha_{2}\right)^{-w_{1}}, \ldots, z_{r} x^{u_{r}}\left(x^{t_{1}}+\alpha_{1}\right)^{-v_{r}}\left(x^{t_{2}}+\alpha_{2}\right)^{-w_{r}}\right]\right\} \\
& =\alpha_{1}^{\sigma} \alpha_{2}^{\mu} x^{\rho-\nu} \sum_{l_{1}, l_{2}=0}^{\infty} \sum_{k=0}^{[n / m]} \sum_{k^{\prime}=0}^{\left[n^{\prime} / m^{\prime}\right]} \frac{(-n)_{m k}\left(-n^{\prime}\right)_{m^{\prime} k^{\prime}}}{k ! k^{\prime} ! l_{1} ! l_{2} !} \\
& A_{n, k} A_{n^{\prime}, k^{\prime}}^{\prime} e^{k} e^{\prime k^{\prime}} \alpha_{1}^{v k+v^{\prime} k^{\prime}} \alpha_{2}^{w k+w^{\prime} k^{\prime}} x^{u k+u^{\prime} k^{\prime}}\left(-\frac{x^{t_{1}}}{\alpha_{1}}\right)^{l_{1}}\left(-\frac{x^{t_{2}}}{\alpha_{2}}\right)^{l_{2}}
\end{aligned}
$$

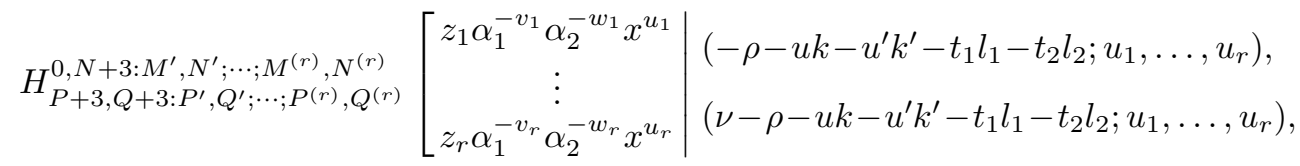

$$
\begin{aligned}
& \left(1+\sigma+v k+v^{\prime} k^{\prime}-l_{1} ; v_{1}, \ldots, v_{r}\right),\left(1+\mu+w k+w^{\prime} k^{\prime}-l_{2} ; w_{1}, \ldots, w_{r}\right), \\
& \left(1+\sigma+v k+v^{\prime} k^{\prime} ; v_{1}, \ldots, v_{r}\right),\left(1+\mu+w k+w^{\prime} k^{\prime} ; w_{1}, \ldots, w_{r}\right), \\
& \left.\left(a_{j} ; \alpha_{j}^{\prime}, \ldots, \alpha_{j}^{(r)}\right)_{1, P}:\left(c_{j}^{\prime}, \gamma_{j}^{\prime}\right)_{1, P^{\prime}} ; \cdots ;\left(c_{j}^{(r)}, \gamma_{j}^{(r)}\right)_{1, P^{(r)}}\right] \\
& \left(b_{j} ; \beta_{j}^{\prime}, \ldots, \beta_{j}^{(r)}\right)_{1, Q}:\left(d_{j}^{\prime}, \delta_{j}^{\prime}\right)_{1, Q^{\prime}} ; \cdots ;\left(d_{j}^{(r)}, \delta_{j}^{(r)}\right)_{1, Q^{(r)}}
\end{aligned}
$$

provided that

(i) $\operatorname{Re}(\nu)<0$; the quantities $t_{1}, t_{2}, u, v, w, u^{\prime}, v^{\prime}, w^{\prime}, u_{1}, v_{1}, w_{1}, \ldots, u_{r}, v_{r}, w_{r}$ are all positive (some of them may however decrease to zero provided that the resulting integral has a meaning); $\max \left\{\left|\arg \left(x^{t_{1}} / \alpha_{1}\right)\right|,\left|\arg \left(x^{t_{2}} / \alpha_{2}\right)\right|\right\}<\pi$.

(ii) $\operatorname{Re}(\rho)+\sum_{i=1}^{r} u_{i} \min _{1 \leq j \leq M^{(i)}}\left[\operatorname{Re}\left(d_{j}^{(i)} / \delta_{j}^{(i)}\right)\right]+1>0$.

(iii) The series occurring on the right-hand side of (7) are absolutely convergent.

It may be noted that Srivastava and Hussain [14] have studied the fractional-derivative formulas without the polynomial products in the interesting paper referred above.

Since the product of an $r$-variable $H$-function and an $s$-variable $H$-funtion is essentially an $(r+s)$-variable $H$-function [5, p.91, Eq.(6)] the FDF 1 is capable of yielding fractional derivative formulae involving product of two or more multivariable $H$-functions.

Fractional derivative formula 2

$$
\begin{aligned}
& D_{x}^{\nu} D_{y}^{\nu^{\prime}}\left\{x^{\rho} y^{\rho^{\prime}}\left(x^{t_{1}}+\alpha_{1}\right)^{\sigma}\left(y^{t_{1}^{\prime}}+\beta_{1}\right)^{\sigma^{\prime}}\left(x^{t_{2}}+\alpha_{2}\right)^{\mu}\left(y^{t_{2}^{\prime}}+\beta_{2}\right)^{\mu^{\prime}}\right. \\
& S_{n}^{m}\left[e x^{u} y^{\lambda}\left(x^{t_{1}}+\alpha_{1}\right)^{v}\left(y^{t_{1}^{\prime}}+\beta_{1}\right)^{\eta}\left(x^{t_{2}}+\alpha_{2}\right)^{w}\left(y^{t_{2}^{\prime}}+\beta_{2}\right)^{\zeta}\right] \\
& S_{n^{\prime}}^{m^{\prime}}\left[e^{\prime} x^{u^{\prime}} y^{\lambda^{\prime}}\left(x^{t_{1}}+\alpha_{1}\right)^{v^{\prime}}\left(y^{t_{1}^{\prime}}+\beta_{1}\right)^{\eta^{\prime}}\left(x^{t_{2}}+\alpha_{2}\right)^{w^{\prime}}\left(y^{t_{2}^{\prime}}+\beta_{2}\right)^{\zeta^{\prime}}\right] \\
& H\left[z_{1} x^{u_{1}} y^{u_{1}^{\prime}}\left(x^{t_{1}}+\alpha_{1}\right)^{-v_{1}}\left(y^{t_{1}^{\prime}}+\beta_{1}\right)^{-v_{1}^{\prime}}\left(x^{t_{2}}+\alpha_{2}\right)^{-w_{1}}\left(y^{t_{2}^{\prime}}+\beta_{2}\right)^{-w_{1}^{\prime}}, \ldots,\right. \\
& \left.\left.z_{r} x^{u_{r}} y^{u_{r}^{\prime}}\left(x^{t_{1}}+\alpha_{1}\right)^{-v_{r}}\left(y^{t_{1}^{\prime}}+\beta_{1}\right)^{-v_{r}^{\prime}}\left(x^{t_{2}}+\alpha_{2}\right)^{-w_{r}}\left(y^{t_{2}^{\prime}}+\beta_{2}\right)^{-w_{r}^{\prime}}\right]\right\} \\
= & \alpha_{1}^{\sigma} \alpha_{2}^{\mu} \beta_{1}^{\sigma^{\prime}} \beta_{2}^{\mu^{\prime}} x^{\rho-\nu} y^{\rho^{\prime}-\nu^{\prime}} \sum_{l_{1}, l_{2}, l_{3}, l_{4}=0}^{\infty} \sum_{k=0}^{[n / m]} \sum_{k^{\prime}=0}^{\left.n^{\prime} / m^{\prime}\right]} \frac{(-n)_{m k}\left(-n^{\prime}\right)_{m^{\prime} k^{\prime}}}{k ! k^{\prime} ! l_{1} ! l_{2} ! l_{3} ! l_{4} !}
\end{aligned}
$$




$$
\begin{aligned}
& A_{n, k} A_{n^{\prime}, k^{\prime}}^{\prime} e^{k} e^{\prime k^{\prime}} \alpha_{1}^{v k+v^{\prime} k^{\prime}} \alpha_{2}^{w k+w^{\prime} k^{\prime}} \beta_{1}^{\eta k+\eta^{\prime} k^{\prime}} \beta_{2}^{\zeta k+\zeta^{\prime} k^{\prime}} x^{u k+u^{\prime} k^{\prime}} y^{\lambda k+\lambda^{\prime} k^{\prime}} \\
& \left(-\frac{x^{t_{1}}}{\alpha_{1}}\right)^{l_{1}}\left(-\frac{x^{t_{2}}}{\alpha_{2}}\right)^{l_{2}}\left(-\frac{y^{t_{1}^{\prime}}}{\beta_{1}}\right)^{l_{3}}\left(-\frac{y^{t_{2}^{\prime}}}{\beta_{2}}\right)^{l_{4}} \\
& H_{P+6, Q+6: P^{\prime}, Q^{\prime} ; \cdots ; P^{(r)}, Q^{(r)}}^{0, N+6: M^{\prime}, N^{\prime} ; \cdots ; M^{(r)}, N^{(r)}}\left[\begin{array}{cc}
z_{1} \alpha_{1}^{-v_{1}} \alpha_{2}^{-w_{1}} \beta_{1}^{-v_{1}^{\prime}} \beta_{2}^{-w_{1}^{\prime}} x^{u_{1}} y^{u_{1}^{\prime}} \\
\vdots \\
z_{r} \alpha_{1}^{-v_{r}} \alpha_{2}^{-w_{r}} \beta_{1}^{-v_{r}^{\prime}} \beta_{2}^{-w_{r}^{\prime}} x^{u_{r}} y^{u_{r}^{\prime}}
\end{array} \mid\right. \\
& \left(-\rho-u k-u^{\prime} k^{\prime}-t_{1} l_{1}-t_{2} l_{2} ; u_{1}, \ldots, u_{r}\right),\left(1+\sigma+v k+v^{\prime} k^{\prime}-l_{1} ; v_{1}, \ldots, v_{r}\right), \\
& \left(\nu-\rho-u k-u^{\prime} k^{\prime}-t_{1} l_{1}-t_{2} l_{2} ; u_{1}, \ldots, u_{r}\right),\left(1+\sigma+v k+v^{\prime} k^{\prime} ; v_{1}, \ldots, v_{r}\right) \text {, } \\
& \left(1+\mu+w k+w^{\prime} k^{\prime}-l_{2} ; w_{1}, \ldots, w_{r}\right),\left(-\rho^{\prime}-\lambda k-\lambda^{\prime} k^{\prime}-t_{1}^{\prime} l_{3}-t_{2}^{\prime} l_{4} ; u_{1}^{\prime}, \ldots, u_{r}^{\prime}\right), \\
& \left(1+\mu+w k+w^{\prime} k^{\prime} ; w_{1}, \ldots, w_{r}\right),\left(\nu^{\prime}-\rho^{\prime}-\lambda k-\lambda^{\prime} k^{\prime}-t_{1}^{\prime} l_{3}-t_{2}^{\prime} l_{4} ; u_{1}^{\prime}, \ldots, u_{r}^{\prime}\right), \\
& \left(1+\sigma^{\prime}+\eta k+\eta^{\prime} k^{\prime}-l_{3} ; v_{1}^{\prime}, \ldots, v_{r}^{\prime}\right),\left(1+\mu^{\prime}+\zeta k+\zeta^{\prime} k^{\prime}-l_{4} ; w_{1}^{\prime}, \ldots, w_{r}^{\prime}\right), \\
& \left(1+\sigma^{\prime}+\eta k+\eta^{\prime} k^{\prime} ; v_{1}^{\prime}, \ldots, v_{r}^{\prime}\right),\left(1+\mu^{\prime}+\zeta k+\zeta^{\prime} k^{\prime} ; w_{1}^{\prime}, \ldots, w_{r}^{\prime}\right), \\
& \left.\left(a_{j} ; \alpha_{j}^{\prime}, \ldots, \alpha_{j}^{(r)}\right)_{1, P}:\left(c_{j}^{\prime}, \gamma_{j}^{\prime}\right)_{1, P^{\prime}} ; \cdots ;\left(c_{j}^{(r)}, \gamma_{j}^{(r)}\right)_{1, P^{(r)}}\right] \\
& \left(b_{j} ; \beta_{j}^{\prime}, \ldots, \beta_{j}^{(r)}\right)_{1, Q}:\left(d_{j}^{\prime}, \delta_{j}^{\prime}\right)_{1, Q^{\prime}} ; \cdots ;\left(d_{j}^{(r)}, \delta_{j}^{(r)}\right)_{1, Q^{(r)}}
\end{aligned}
$$

provided that

(i) $\operatorname{Re}(\nu)<0, \operatorname{Re}\left(\nu^{\prime}\right)<0$; the quantities $t_{1}, t_{2}, t_{1}^{\prime}, t_{2}^{\prime}, u, v, w, \lambda, \eta, \zeta, u^{\prime}, v^{\prime}, w^{\prime}, \lambda^{\prime}, \eta^{\prime}$, $\zeta^{\prime}, u_{1}, v_{1}, w_{1}, \ldots, u_{r}, v_{r} w_{r}, u_{1}^{\prime}, v_{1}^{\prime}, w_{1}^{\prime}, \ldots, u_{r}^{\prime}, v_{r}^{\prime}, w_{r}^{\prime}$ are all positive (some of them may however decrease to zero provided that the resulting integral has a meaning); $\max \left\{\left|\arg \left(x^{t_{1}} / \alpha_{1}\right)\right|,\left|\arg \left(x^{t_{2}} / \alpha_{2}\right)\right|,\left|\arg \left(y^{t_{1}^{\prime}} / \beta_{1}\right)\right|,\left|\arg \left(y^{t_{2}^{\prime}} / \beta_{2}\right)\right|\right\}<\pi$.

(ii) $\operatorname{Re}(\rho)+\sum_{i=1}^{r} u_{i} \min _{1 \leq j \leq M^{(i)}}\left[\operatorname{Re}\left(d_{j}^{(i)} / \delta_{j}^{(i)}\right)\right]+1>0$ and $\operatorname{Re}\left(\rho^{\prime}\right)+\sum_{i=1}^{r} u_{i}^{\prime} \min _{1 \leq j \leq M^{(i)}}\left[\operatorname{Re}\left(d_{j}^{(i)} / \delta_{j}^{(i)}\right)\right]+1>0$.

(iii) The series occuring on the right-hand side of (8) are absolutely convergent.

Proofs of FDF 1 and FDF 2. To prove the fractional derivative formula (FDF) 1, we first express both the general class of polynomials $S_{n}^{m}$ and $S_{n^{\prime}}^{m^{\prime}}$ occurring on its left-hand side in the series form given by (3) and replace the multivariable $H$-function occurring therein by its well known Mellin-Barnes contour integral given by (4). Now making use of the following binomial expansions

$$
\begin{aligned}
& \left(x^{t_{1}}+\alpha_{1}\right)^{\sigma}=\alpha_{1}^{\sigma} \sum_{l_{1}=0}^{\infty}\left(\begin{array}{l}
\sigma \\
l_{1}
\end{array}\right)\left(\frac{x^{t_{1}}}{\alpha_{1}}\right)^{l_{1}}, \quad\left|x^{t_{1}} / \alpha_{1}\right|<1 \\
& \left(x^{t_{2}}+\alpha_{2}\right)^{\mu}=\alpha_{2}^{\mu} \sum_{l_{2}=0}^{\infty}\left(\begin{array}{c}
\mu \\
l_{2}
\end{array}\right)\left(\frac{x^{t_{2}}}{\alpha_{2}}\right)^{l_{2}}, \quad\left|x^{t_{2}} / \alpha_{2}\right|<1
\end{aligned}
$$


and using the formula [8, p.67, Eq.(4.4.4)]

$$
D_{x}^{\nu}\left\{x^{\rho}\right\}=\frac{\Gamma(\rho+1)}{\Gamma(\rho-\nu+1)} x^{\rho-\nu}, \quad \operatorname{Re}(\rho)>-1 .
$$

We easily arrive at the desired formula FDF 1 after a little simplification.

The FDF 2 can be obtained on using the FDF 1 twice, first with respect to the variable $y$, and then with respect to the variable $x$; here $x$ and $y$ are independent variables.

\section{Special Cases and Applications}

The fractional derivative formulae 1 and 2 established here are unified in nature and act as key formulae. Thus the general class of polynomials involved in the FDF 1 and 2 reduce to a large number of polynomials listed by Srivastava and Singh [16, pp.158-161], therefore from the formulae 1 and 2 we can further obtain various fractional derivative formulae involving a number of simpler polynomials. Again, the multivariable $H$-function occurring in these formulae can be suitably specialized to a remarkably wide variety of useful functions (or product of several such functions) which are expressible in terms of $E$, $F, G$ and $H$-functions of one, two or more variables. For example, if $N=P=Q=0$, the multivariable $H$-function occurring in the left-hand side of these formulae would reduce immediately to the product of $r$ different $H$-functions of Fox [2]; thus the table listing various special cases of the $H$-function [7, pp.145-159] can be used to derive from these fractional derivative formulae a number of othere FDF involving any of these simpler special functions.

On making suitable specializations in the parameters involved in our polynomials and the multivariable $H$-function we get in essence the results obtained by Srivastava, Chandel and Vishwakarma [11, pp.563-564, Eqs.(2.1) and (2.3)], Srivastava and Goyal [12, pp.644-645, Eqs.(2.1) and (2.3)], Gupta, Agrawal and Soni [4, pp.71-72, Eq.(2.1)] and Gupta and Agrawal [3, p.171, Eq.(3.1)].

If in the FDF 1 , we reduce the polynomial $S_{n}^{m}$ to the Konhauser biorthogonal polynomials [10, p.225, Eq.(3.23); 6, p.304, Eq.(5)] the polynomial $S_{n^{\prime}}^{m^{\prime}}$ to the Jacobi polynomials [16, p.159, Eq.(1.6)] and the multivariable $H$-function to the product of $r$ different modified Bessel functions of the second king [13, p.18, Eq.(2.6.6)] we arrive at the following new and interesting special case of the FDF 1 after a little simplification

$$
\begin{aligned}
& D_{x}^{\nu}\left\{x^{\rho+\frac{r}{2}}\left(x^{t_{1}}+\alpha_{1}\right)^{\sigma}\left(x^{t_{2}}+\alpha_{2}\right)^{\mu} Z_{n}^{\alpha}\left(x^{\frac{1}{\beta}} ; \beta\right) P_{n^{\prime}}^{(\delta, \tau)}[1-2 x] \prod_{i=1}^{r} K_{\nu_{i}}\left[z_{i} x\right]\right\} \\
= & 2^{-\frac{2 r}{3}} \prod_{i=1}^{r}\left(z_{i}\right)^{-\frac{1}{2}} \alpha_{1}^{\sigma} \alpha_{2}^{\mu} x^{\rho-v} \sum_{l_{1}, l_{2}=0}^{\infty} \sum_{k=0}^{n} \sum_{k^{\prime}=0}^{n^{\prime}} \frac{(-n)_{k}\left(-n^{\prime}\right)_{k^{\prime}}}{k ! k^{\prime} ! l_{1} ! l_{2} !} \frac{\Gamma(1+\alpha+\beta n)}{n ! \Gamma(1+\alpha+\beta k)}\left(\begin{array}{c}
n^{\prime}+\delta \\
n^{\prime}
\end{array}\right) \\
& \frac{\left(\delta+\tau+n^{\prime}+1\right)_{k^{\prime}}}{(\delta+1)_{k^{\prime}}} x^{k+k^{\prime}}\left(-\frac{x^{t_{1}}}{\alpha_{1}}\right)^{l_{1}}\left(-\frac{x^{t_{2}}}{\alpha_{2}}\right)^{l_{2}} \frac{\Gamma\left(l_{1}-\sigma\right) \Gamma\left(l_{2}-\mu\right)}{\Gamma(-\sigma) \Gamma(-\mu)} \\
& H_{1,1: 0,2 ; \cdots ; 0 ; 2}^{0,1: 2, \cdots ; 2,0}\left[\begin{array}{c}
z_{1} x \\
\vdots \\
z_{r} x
\end{array} \mid \begin{array}{ll}
\left(-\rho-k-k^{\prime}-t_{1} l_{1}-t_{2} l_{2} ; 1, \ldots, 1\right) \quad: \quad-\quad ; \cdots ; \quad- \\
\left(\nu-\rho-k^{\prime}-t_{1} l_{1}-t_{2} l_{2} ; 1, \ldots, 1\right):\left(\frac{1}{4} \pm \frac{\nu_{1}}{2}, \frac{1}{2}\right) ; \cdots ;\left(\frac{1}{4} \pm \frac{\nu_{r}}{2}, \frac{1}{2}\right)
\end{array}\right]
\end{aligned}
$$


The conditions of validity of (10) can be easily obtained from those of (7). Further, on taking $\beta=1$ in (10), the Konhauser biorthogonal polynomials occurring therein reduces to the Laguerre polynomials and we get the corresponding FDF involving the Laguerre polynomials $L_{n}^{(\alpha)}(x)$.

Several othere interesting and useful special cases of our main fractional derivative formulae 1 and 2 involving the product of a large variety of polynomials (which are special cases of $S_{n}^{m}$ and $S_{n^{\prime}}^{m^{\prime}}$ ) and numerous simple special functions involving one or more variables (which are particular cases of the multivariable $H$-function) can also be obtained but we do not record them here for lack of space.

\section{References}

[1] A. Erdélyi, W. Magnus, F. Oberhettinger and F. G. Tricomy, Tables of Integral Transforms, Vol.II. McGraw-Hill, New York, 1954.

[2] C. Fox, The $G$ and $H$-functions as symmetrical Fourier kernels, Trans. Amer. Math. Soc. 98(1961), 395-429.

[3] K. G. Gupta and S. M. Agrawal, Fractional integeral formulae involving a general class of polynomials and the multivariable $H$-function, Proc. Indian Acad. Sci. (Math. Sci.) 99 (1989), 169-173.

[4] K. C. Gupta and S. M. Agrawal and R. C. Soni, Fractional integral formulae involving the multivariable $H$-function and a general class of polynomials, Indian J. Pure Appl. Math., 21(1990), 70-77.

[5] K. C. Gupta and R. C. Soni, A study of H-functions of one and several variables, J. Rajasthan Acad. Phy. Sci. 1(2002), 89-94.

[6] J. D. E. Konhauser, Biorthogonal polynomials suggested by the Lagurre polynomials, Pacific J. Math. 21(1967), 303-314.

[7] A. M. Mathai and R. K. Saxena, The $H$-Function with Applications in Statistics and Other Disciplines, Wiley Eastern Limited, New Delhi, 1978.

[8] K. B. Oldham and J. Spanier, The Fractional Calculus, Academic Press, New York, 1974.

[9] H. M. Srivastava, A contour integral involving Fox's H-function, Indian J. Math. 14(1972), $1-6$.

[10] H. M. Srivastava, The Weyl fractional integral of a general class of polynomials, Boll. Unione Mat. Ital. 2-B(1983), 219-228.

[11] H. M. Srivastava, R. C. Singh Chandel and P. K. Vishwakarma, Fractional derivatives of certain generalized hypergeometric functions of several variables, J. Math. Anal. Appl., 184(1994), 560-572.

[12] H. M. Srivastava and S. P. Goyal, Fractional derivatives of the $H$-function of several variables, J. Math. Anal. Appl. 112 (1985), 641-651.

[13] H. M. Srivastava, K. C. Gupta and S. P. Goyal, The $H$-Fucntions of One and Two Variables with Applications, South Asian Publishers, New Delhi, 1982.

[14] H. M. Srivastava and M. A. Hussain, Fractional integration of the $H$-function of several variables, Comput. Math. Appl. 30(1995), 73-85.

[15] H. M. Srivastava and R. Panda, Some bilateral generating functions for a class of generalized hypergeometric polynomials, J. Reine Angew Math. 283/284(1976), 265-274.

[16] H. M. Srivastava and N. P. Singh, The integration of certain products of the multivariable $H$-function with a general class of polynomials, Rend. Circ. Mat. Palermo. 32(1983), 157 $-187$.

Department of Mathematics, M. N. Institute of Technology, Jaipur 302017, India.

E-mail: drrcsoni@yahoo.com 